“C 2016 IEEE. Personal use of this material is permitted. Permission from IEEE must be obtained for all other uses, in any current or future media, including reprinting/republishing this material for advertising or promotional purposes, creating new collective works, for resale or redistribution to servers or lists, or reuse of any copyrighted component of this work in other works." 


\section{Comparing Effectiveness of Hybrid MPPT Algorithms under Partial Shading Conditions}

Mohammad R. Al-Soeidat

Student Member, IEEE,

School of Electrical, Mechanical and Mechatronic Systems, University of Technology, Sydney, Australia mohammad.r.al-

soeidat@student.uts.edu.au

\author{
Alexis Cembrano \\ School of Electrical and \\ Information Engineering, The \\ University of Sydney, Australia \\ acem3575@uni.sydney.edu.au
}

\author{
Dylan D-C. Lu, SMIEEE \\ ${ }^{1}$ School of EMMS, University of \\ Technology, Sydney, Australia \\ ${ }^{2}$ School of EIE, The University of \\ Sydney, NSW 2006, Australia \\ dylan.lu@uts.edu.au
}

\begin{abstract}
For photovoltaic (PV) systems, a key area which can affect the amount of energy harvested is the effectiveness of the Maximum Power Point Tracking (MPPT) algorithm, which dynamically locates the operating point for maximum power output. Currently, there are many MPPT algorithms proposed and in use, however, they are not without problems. For example, algorithms such as the Fractional Open Circuit method are simple and effective, but their accuracy is poor. Other algorithms such as the Perturb \& Observe (P\&O) approach, although more accurate, will cause oscillations around the maximum power point. It is perceived that the use of two MPPT algorithms in tandem will help to overcome the drawbacks of individual MPPT algorithms used in isolation. This paper proposes two new versions of Hybrid MPPT algorithm; one being a combination of the Fractional Open Circuit Voltage and P\&O methods, and the other a combination of the Power Increment and P\&O techniques. Experimental results are reported to evaluate and compare the performance of the algorithms.
\end{abstract}

Index Terms-Hybrid algorithm, maximum power point tracking (MPPT), partial shading, photovoltaic (PV).

\section{INTRODUCTION}

$\mathrm{T}$ HE Maximum Power Point Tracking (MPPT) algorithm is typically implemented by a controller for the PV panel's power converter, dynamically locating the operating point which delivers maximum power output. This is of particular importance when the PV array becomes partially shaded. Fig. 1 shows this operating point on the characteristic I-V and P-V curves.
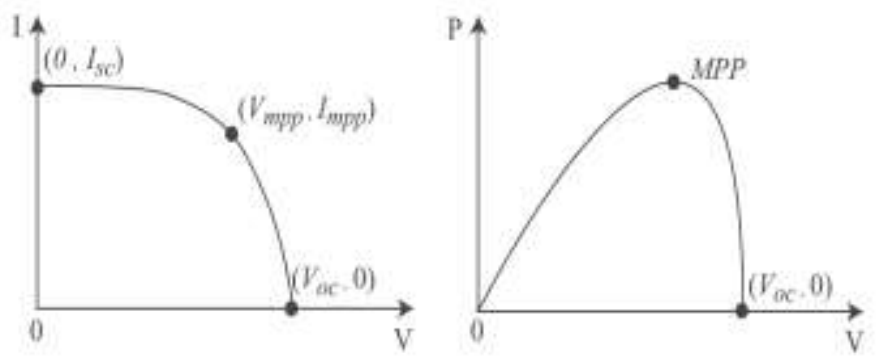

Fig. 1. Characteristic curves of a PV array [1].
However, under partial shading conditions, these characteristic curves are shaped quite differently, as shown in Fig. 2. At Fig. 2(b) and Fig 2(c) we see the I-V and the P-V curves respectively for a partially shaded $3 \times 2 \mathrm{PV}$ array. In this case, one out of the six PV modules (Fig. 2(a)) is shaded.

It can be noted that when the PV array undergoes partial shading, numerous peaks or maxima lower than the maximum operating point are created, usually depending on the number

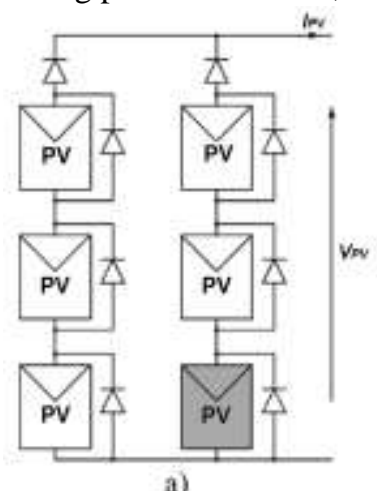

a)
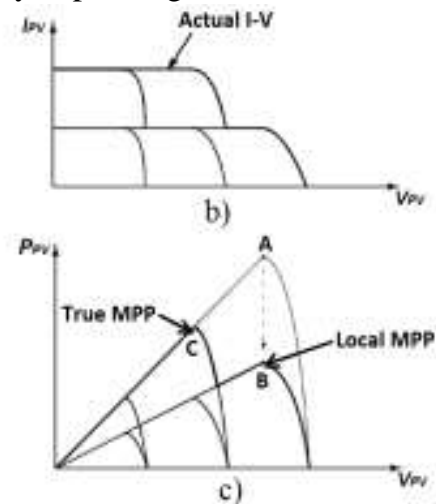

Fig. 2. Partial shading of $3 \times 2 \mathrm{PV}$ array (a), corresponding I-V curve (b), corresponding P-V curve (c).

of PV modules which are shaded [3]. These peaks are typically referred to as "local maxima", while the largest peak or highest operating point is usually referred to as the "global" or "true" maximum operating point. These conditions present difficulties for single MPPT algorithms, and can greatly affect their accuracy, as single algorithms are usually optimized for unshaded conditions only.

Some single MPPT algorithms currently used are P\&O, Incremental Conductance, Fractional Open Circuit Voltage, Fractional Short Circuit Current, and the Power Increment Technique. However, most of these have been designed to operate under uniform environmental conditions, and the maximum power which they can extract can decrease considerably under quickly changing partial shading conditions (PSC) [4]. There are also other limitations associated with each of these methods, such as improving the MPP tracking speed to the detriment of its accuracy. 
In this paper, two hybrid MPPT algorithms aiming at improving partial shading tracking performance are proposed. The paper is organized as follows: In Section II the problems with different single MPPT algorithm against PSC are discussed. It follows by the introduction and analysis of the two proposed hybrid algorithms. The experimental setup and results are explained and shown in Sections III and IV respectively, followed by the conclusion in Section V.

\section{PROPOSED HYBRID MAXIMUM POWER POINT TRACKING ALGORITHMS}

Several conventional single MPPT algorithms were chosen for observation and comparison, noting the known advantages and disadvantages. Following their brief discussion, the proposed hybrid MPPT algorithms are covered in detail. These seek to overcome the disadvantages identified with the single MPPT algorithms.

\section{A. Single MPPT Algorithms}

The first method is the Power Increment technique. In this method, the power converter is controlled so as to draw power in a successive manner, starting from the open circuit voltage condition (point B1 on P-V curve, Fig. 3).

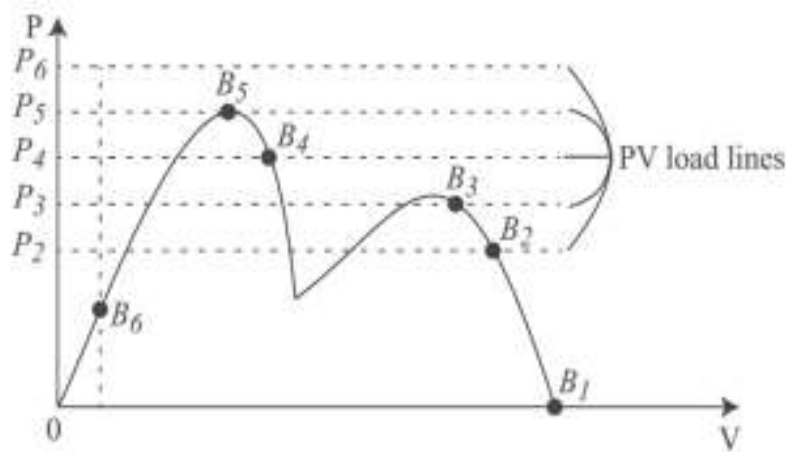

Fig. 3. Illustration of the constant power lines of the Power Increment Technique [3].

The voltage is varied across the panel's entire operating range, and the measured power levels are recorded and stored at intervals, indicated as the points B2 to B6 in Fig. 3. The maximum power point is then identified as B5, and then this is set as the new operating point of the system [3].

The biggest advantage of this method is that it can differentiate between the local and global maxima, meaning that it is suitable as an MPPT technique for PV panels subject to frequently changing partial shading conditions.

However, its accuracy is dependent on the resolution of the recorded power levels, and is also typically slower than other methods such as Fractional Open Circuit Voltage.

The second method is the Fractional Open Circuit Voltage technique. It utilizes the fact that there is a linear relationship between the PV panel's open circuit voltage (Voc) and the maximum power point voltage $\left(\mathrm{V}_{\mathrm{MPP}}\right)$ for the different irradiance levels on the PV panel [7]. The linear relationship can be described as follows:

$$
\mathrm{V}_{\mathrm{MPP}}=\mathrm{k} 1 \mathrm{Voc}
$$

The parameter k1 is a proportional constant, usually found to be between 0.71 and 0.78 , dependent on the construction and physical characteristics of the particular model of PV panel.

After sensing the open circuit voltage at a particular instant, the controller will adjust its duty cycle and hence the system's operating point until the output voltage of the PV panel matches the predicted $\mathrm{V}_{\text {MPP. }}$

The advantage of this method is that it is a typically reliable method of quickly reaching the vicinity of the maximum power operating point.

The disadvantages are that it can only offer a low accuracy when trying to reach the precise location of the MPP, and this is also because a change to the irradiance levels on the PV array will require a recalculation of $V_{M P P}$. Whenever the algorithm is performed, a measurement of the open circuit voltage needs to be made, and the system needs to be stopped in order to make the measurement, thereby necessitating a temporary loss of power.

The third method is the Perturb and Observe (P\&O) technique. With each cycle, this method perturbs the system's operating point by either perturbing the PV panel current, or by perturbing the duty cycle of the power converter.

The output power of the two operating points is compared, and if the change in output power is positive (i.e. the power produced at the new operating point is greater), then the controller will make its next perturbation in the same direction. A perturbation in the opposite direction will be made if the output power is negative.

The main advantage of this technique is that it is effective and simple to implement - the hardware requirements are only voltage and current sensors, from which the Power can be calculated [1].

However, it is unable to cope with frequently changing irradiance conditions on the PV panels, which would thereby also change the PV curve frequently. Also, it is susceptible to being trapped in local maxima, as it is unable to distinguish between these and the true MPP of the P-V curve. If the system operating point is located at a local maximum, the operating point will only oscillate backwards and forwards about that location.

\section{B. Hybrid MPPT Algorithms}

By using hybrid MPPT algorithms, the weaknesses of an individual MPPT algorithm under PSC can be overcome by the benefits of another it is combined with. The key issue of distinguishing between local and global MPP can be addressed by combining one algorithm, which detects the vicinity of the "true" or global maximum power operating point, with another algorithm, which has a high level of MPPT accuracy over a smaller range.

Both of the new hybrid MPPT algorithms presented in this paper address the above mentioned issues. The general location of the global maximum is located first by either the 
Fractional Open Circuit Voltage method, or the Power Increment method. These algorithms are then combined with the $\mathrm{P} \& \mathrm{O}$ method to precisely locate the MPP.

Previous methods have been suggested already, such as the Fractional Short Circuit Current with P\&O method [5], which solves the above mentioned problems in similar fashion without the use of sensors. Also, numerous variations of the Fractional Open Circuit Voltage method with $\mathrm{P} \& \mathrm{O}$ method have been previously suggested, but mostly tailored to suit particular applications, such as on wind generators [6].

The hybrid algorithms proposed in this paper utilize traditional voltage and current sensing methods, in combination with an inexpensive microcontroller. The proposed Fractional Open Circuit Voltage with $\mathrm{P} \& \mathrm{O}$ method features refinements in the determination of the proportional constant, and in the accuracy of the $\mathrm{P} \& \mathrm{O}$ section. The design for the Power Increment method with $\mathrm{P} \& \mathrm{O}$ also features similar refinements in its $\mathrm{P} \& \mathrm{O}$ section, with the Power Increment technique being chosen for its ability to thoroughly scan the entire operating range for the MPP.

\section{Fractional Open Circuit with Perturb \& Observe}

The first of the proposed hybrid algorithms is the Fractional Open Circuit Voltage with P\&O, shown at Fig. 4.

The algorithm starts by measuring the open circuit voltage of the PV system, by setting the duty cycle of the buck converter to zero (thereby the buck converter acts as an open circuit). MPP voltage is then calculated by multiplying the $\mathrm{k}$ factor with the measured open circuit voltage. Then, the duty cycle of the buck converter starts to sweep until the output voltage of the PV system equals the calculated Vmpp. Then, the duty cycle of the buck converter is fixed at that value.

The second stage of the hybrid algorithm is the P\&O method. With each iteration of this algorithm, an initial measurement of the PV Panel's output voltage and current is made, and their values multiplied in order to calculate the PV panel output power. The output power of the new operating point is then compared with the output power at the previous operating point.

If the change in output power is positive (i.e. the power produced at the new operating point is greater), then the controller will make its next perturbation in the same direction.

If the change in output power is negative (i.e. the power produced at the new operating point is less), then the controller will make the next perturbation so as to move the operating point in the opposite direction.

The P\&O loop continues to cycle 25 iterations, and when this is completed, a check will be made to see if a significant insolation change had occurred since starting the $25 \mathrm{P} \& \mathrm{O}$ iterations. This is accomplished by comparing the current operating point with the one prior to starting the $25 \mathrm{P} \& \mathrm{O}$ iterations, and a significant insolation change is confirmed when there is more than $15 \%$ change in output voltage.

If significant insolation change is detected, the algorithm will return to the beginning, and start by determining the new open circuit voltage.
Under the partial shading arrangement, it was experimentally found that the proportional constant was close to 0.45 , much less than the PV panel's experimentally measured value of around 0.755 under unshaded conditions, thus highlighting a key problem with using the fractional open circuit voltage algorithm under PSC. In order to overcome this problem, the algorithm was designed so that, under PSC (when the measured voltage is less than $85 \%$ of the voltage measured under unshaded conditions), the correct value of $\mathrm{k}$ was acquired by multiplying $\mathrm{k}$ by itself 3 times, i.e., $\mathrm{k}=$ $0.755 * 0.755 * 0.755=0.43$.

\section{Power Increment with Perturb \& Observe $(P \& O)$}

The other hybrid algorithm proposed in this paper is Power Increment with $\mathrm{P} \& \mathrm{O}$, shown at Fig. 5. At the Power increment

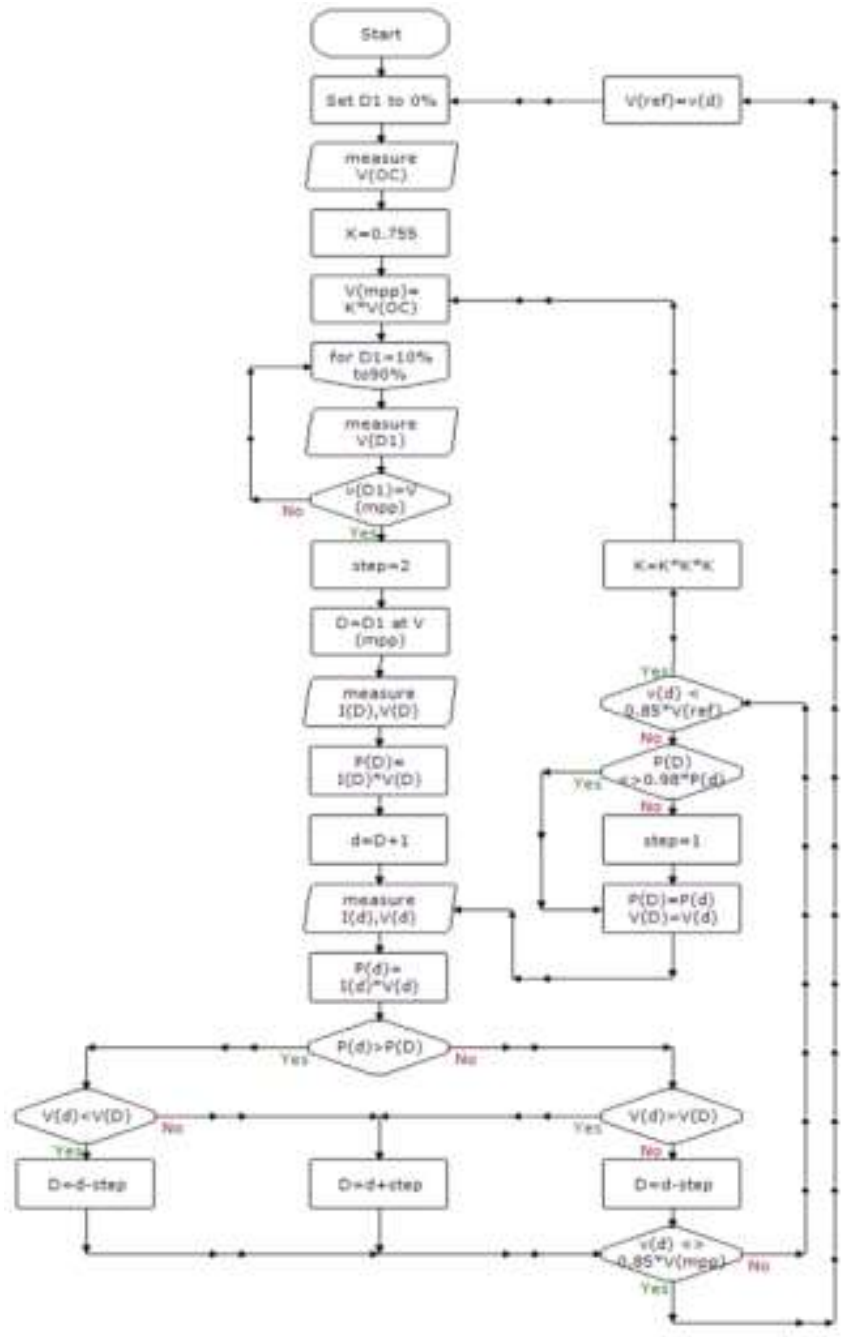

Fig. 4. Flowchart for hybrid algorithm: Fractional Open Circuit Voltage with Perturb and Observe (P\&O).

section of the algorithm, variables such as maximum and minimum duty cycles are firstly initialized so that system limits can be specified. The power converter's duty cycle is initially set to $10 \%$ (the minimum duty cycle for the system), in preparation for scanning the output power over the chosen operating range of duty cycles: $10 \%$ to $90 \%$.

The duty cycle is then increased from $10 \%$ to $90 \%$ over 65 
iterations. With each iteration, the PV panel output voltage and current is measured, and multiplied together to obtain the PV panel's output power. This value is then compared against the previously stored maximum power, and is stored as the new maximum power (with the corresponding duty cycle) if it is larger in value.

After the entire operating range of duty cycles have been scanned, the stored maximum output power is checked against the previously defined limits for output power. If it is found to exceed either of the limits, the output power is clamped appropriately, to ensure the panel remains within its normal range of operation.

The power converter's duty cycle is then changed to the value for which the MPP was found, and the next stage of the hybrid algorithm $(\mathrm{P} \& \mathrm{O})$ is commenced.

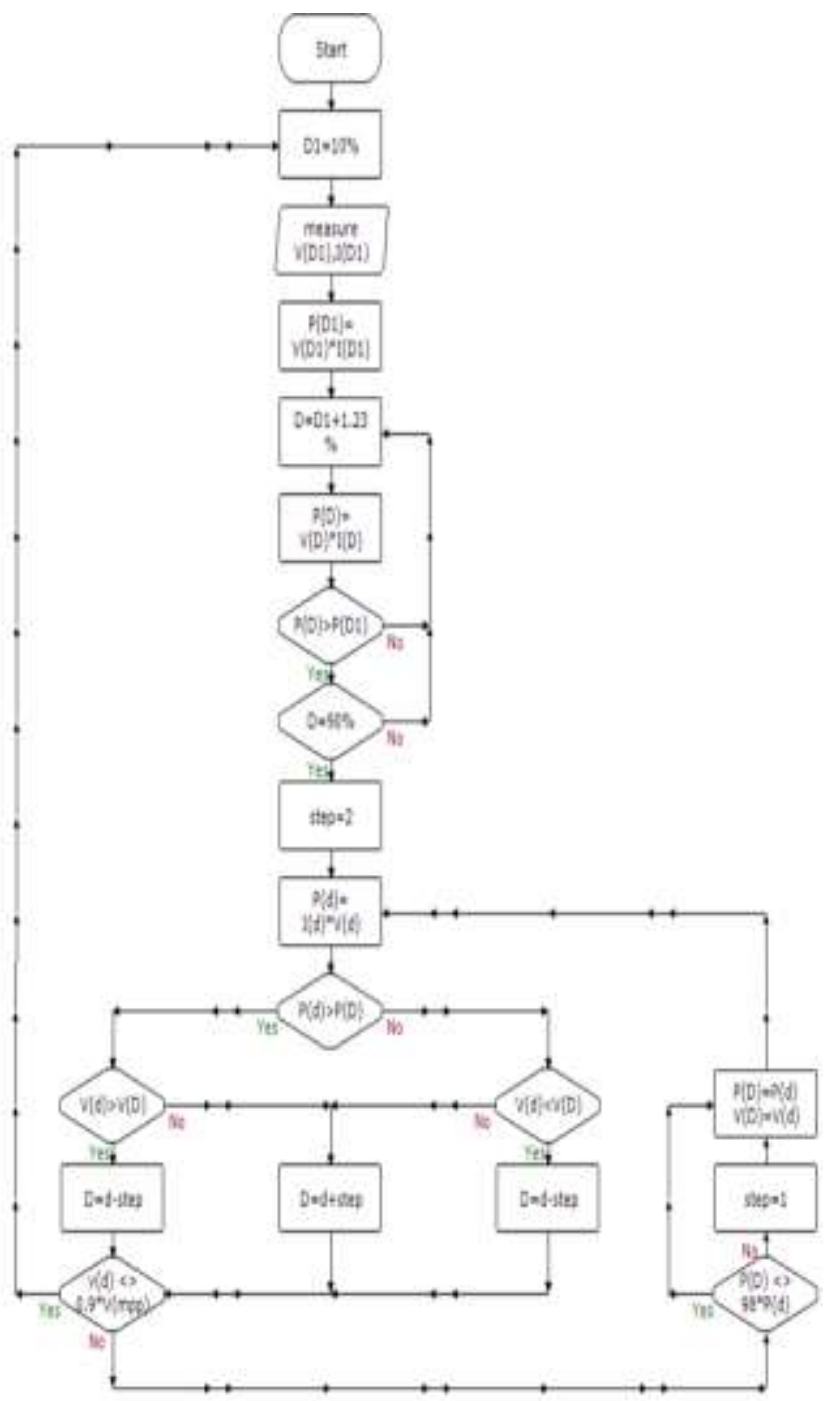

Fig. 5. Flowchart for hybrid algorithm: Power Increment with Perturb and Observe (P\&O)

The process for the P\&O loop is very similar to the one used for the $\mathrm{P} \& \mathrm{O}$ section of the previous hybrid algorithm at Fig. 4. The loop cycles 25 iterations, and when this is completed, a check will be made for significant insolation change. When there is more than $85 \%$ change in output power, the algorithm returns to the Stage 1 section (Power Increment technique) to scan the entire operating range. As before, if a large insolation change is not detected, then the system continues performing the $\mathrm{P} \& \mathrm{O}$ method, restarting to cycle through another 25 iterations.

\section{EXPERIMENTAL SETUP}

Fig. 6 is a block diagram of the implemented system, while Fig. 7 is the corresponding schematic diagram. Initially, in order to record the P-V characteristics of the system, the PV array was connected directly to the resistive load bank.

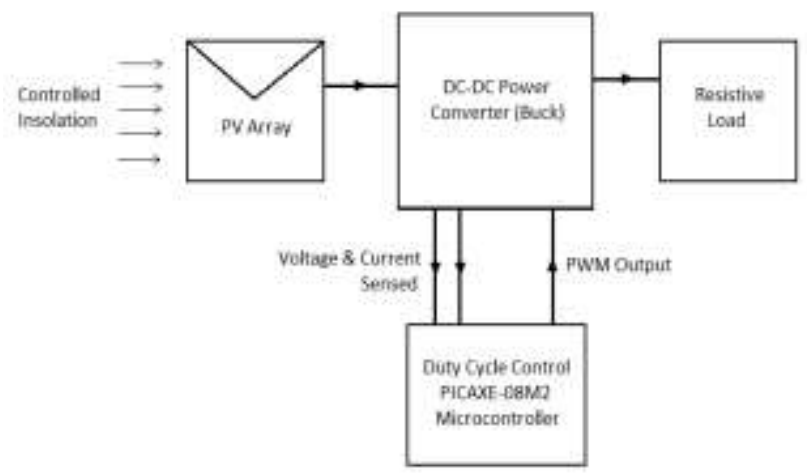

Fig. 6. Block diagram of experimental setup.

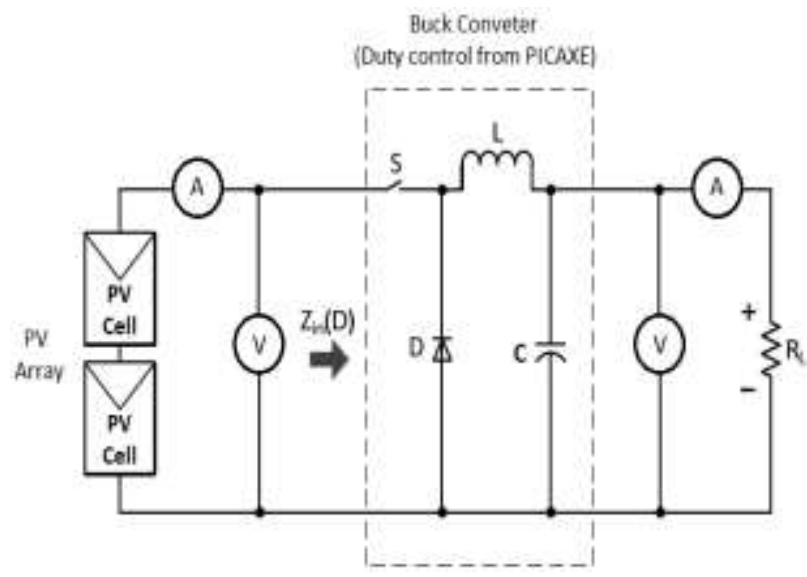

Fig. 7. Schematic diagram of experimental setup.

The PV array consisted of two 10W photovoltaic panels connected in series. Centered in front of each PV panel, one $500 \mathrm{~W}$ halogen lamp was positioned at $320 \mathrm{~mm}$ away from the panel surface. Each panel was arranged so that its surface was as parallel as possible to the corresponding halogen lamp surface, in order to maximise insolation.

The PV array was connected to a $5 \mathrm{~W}$ resistor load bank, whose value could be configured between $1 \mathrm{ohm}$ and 270 ohms. A current and volt meter was used to obtain the P-V characteristic data and P-V curve, firstly under full insolation (no shaded PV cells), and then under a partially shaded condition (half of one PV cell surface was covered).

Experimental equipment was set up inside a laboratory room, pictured at Fig 8. The environment was subject to 
minimal lighting from the outdoors, having a single entry/exit point more than 3 metres away from the equipment setup, and having only very narrow windows positioned very close to the ceiling. These conditions were advantageous, as it minimized the insolation contributed from ambient sources of lighting, and ensured that the majority of the panel insolation originated from the halogen lamps only.

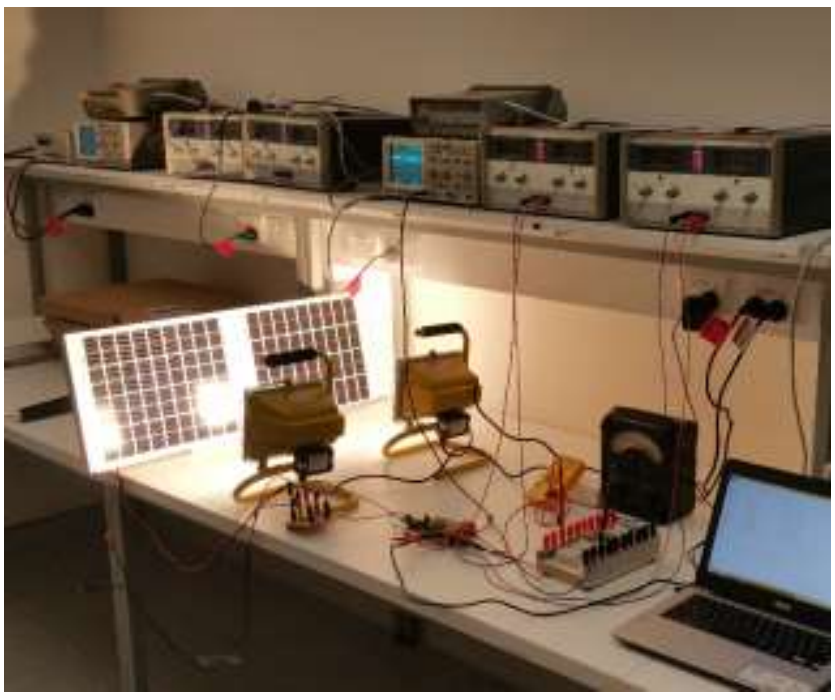

Fig. 8. Environment for laboratory experimentation.

\section{EXPERIMENTAL RESULTS}

With the PV array connected directly to the resistor load bank, voltage and current measurements were made and the data used to construct the P-V and I-V curves for unshaded and partially shaded conditions (Fig. 9 and Fig. 10 respectively). This data acted as a baseline against which the performance of the single and hybrid MPPT algorithms could be experimentally compared.

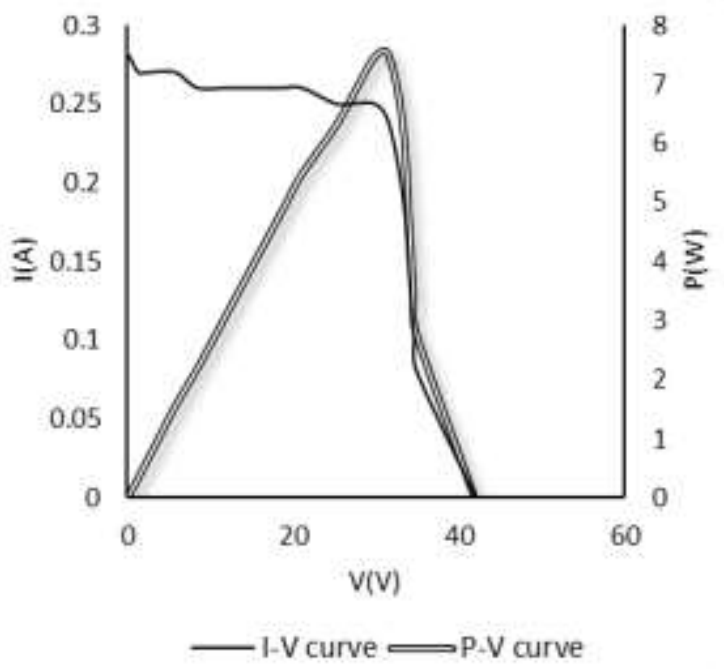

Fig. 11. P-V and I-V curve for 2 unshaded PV panels.

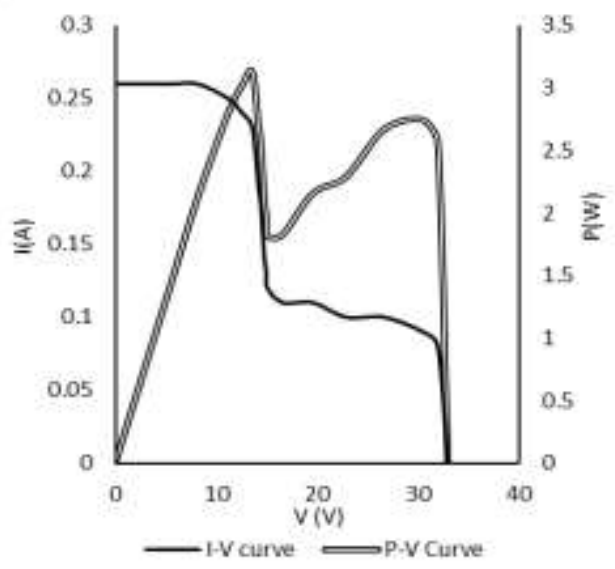

Fig. 12. P-V and I-V curve for 1 unshaded, 1 half-shaded PV panel.

After acquiring the baseline data, the buck power converter was then connected as shown in Fig. 6 and Fig. 7, to implement the proposed hybrid MPPT algorithms. The percentage error was found using formula (1), and then accuracy of each algorithm was found by subtracting the percentage error from 100.

$$
\text { Error } \%=\frac{\text { Power }_{P V(\text { Actual })}-\text { Power }_{P V(\text { Expected })}}{\text { Power }_{P V(\text { Expected })}} * 100 \%
$$

For each algorithm, each test was repeated 5 times under the same environmental conditions, and the average of each result was used. The summary comparison of experimental results for the single and the proposed hybrid MPPT algorithms are provided at Table I.

TABLE I

PERFORMANCE COMPARISON OF SINGLE AND HYBRID MPPT ALGORITHMS

\begin{tabular}{|c|c|c|c|c|}
\hline Aigorithm & Shading & $\begin{array}{l}\text { time to reach } \\
\operatorname{MPP}(s)\end{array}$ & $\begin{array}{l}\text { (\%) Accuracy } \\
\text { detecting Mpp } \\
\text { Duty Cycle }\end{array}$ & $\begin{array}{l}\text { (\%) Accuracy } \\
\text { detecting PV } \\
\text { power at MPp }\end{array}$ \\
\hline \multirow{2}{*}{$\begin{array}{c}\text { Fractional Open Circuit } \\
\text { Voltage } \\
\end{array}$} & unshaded & 7.86 & 20.78 & 80.63 \\
\hline & partial shading & 7.86 & 66.70 & 69.91 \\
\hline \multirow{2}{*}{$\begin{array}{c}\text { Perturb \& Observe } \\
\text { [P\&O] }\end{array}$} & unshaded & 3.04 & $\$ 1.69$ & 89.17 \\
\hline & partial shading & 3.04 & 69.70 & 44.87 \\
\hline \multirow{2}{*}{ Power Increment } & unshaded & 7,36 & 83.07 & 57.40 \\
\hline & partial shading & 7.56 & 77.67 & 69.91 \\
\hline \multirow{2}{*}{$\begin{array}{c}\text { Fractional Open Circuit } \\
\text { Voltage }+P 80\end{array}$} & unshaded & 9.91 & 96.11 & 98.89 \\
\hline & partial shading & 12.91 & 95.16 & 73.75 \\
\hline \multirow{2}{*}{$\begin{array}{l}\text { Power increment + } \\
\text { P\&O }\end{array}$} & unshaded & 9.27 & 95.24 & 86.11 \\
\hline & partial shading & 11.27 & 93.63 & 68.22 \\
\hline
\end{tabular}

In terms of time to detect the MPP, the results for the single algorithms were as expected. $\mathrm{P} \& \mathrm{O}$ was the quickest to complete, but this was under the condition that the MPPT algorithm was positioned to scan close to the vicinity of the real MPP, at the start of the algorithm. In comparison, the Fractional Open Circuit Voltage and Power Increment methods took almost twice as long to complete, since their implementation involved scanning a large if not entire portion of the duty cycle range.

The time to complete the hybrid algorithms were similar to the sum of the times for the original algorithms, although it should be noted a low cost 8-bit microcontroller was used in 
these experiments, hence why the observed scan times were much slower than those typically offered by commercial solutions. Additionally, delays were added throughout the algorithms' code for recording purposes and for greater ease of observations.

In the case of the hybrid Fractional Open Circuit Voltage and $\mathrm{P} \& \mathrm{O}$, this algorithm took around $60 \%$ longer than the single algorithm, while the Power Increment and P\&O took around 49\% longer to complete. This was the expected disadvantage with the hybrid algorithms, however the tradeoff was found in the much improved accuracy in detecting the MPP under partial shading conditions.

The accuracy in detecting the duty cycles under partial shading conditions was improved by between 10 to $20 \%$ when implementing the hybrid algorithms, largely as a result of the single algorithms' inability to adapt to changing conditions of insolation.

It is also important to note that in the experiments, the PV system output voltage and current was found by using a measuring resistor. A large amount of heat was generated at the resistor, indicating a notable amount of consequent power losses, thus explaining the particularly low values of output power detection accuracy observed at Table I. The use of current sensors for the same measurements is recommended in future experimentation.

\section{CONCLUSION}

This paper has proposed and experimentally verified two new hybrid MPPT algorithm variations, which offer significant advantages for MPPT under partial shading conditions. The proposed Fractional Open Circuit Voltage and Power Increment methods are able to effectively detect the vicinity of the maximum power operating point, and then these are combined with the $\mathrm{P} \& \mathrm{O}$ technique to more precisely locate the MPP's exact location. These hybrid algorithms contribute to the improvement of PV panel accuracy and efficiency under changing environmental conditions.

\section{REFERENCES}

[1] A. Bidram, A. Davoudi and S. Balog, "Control and Circuit Techniques to Mitigate Partial Shading Effects in Photovoltaic Arrays", IEEE Journal of Photovoltaics, Vol.2, No.4, Oct. 2012.

[2] H. Patel and V. Argawal, "MATLAB-Based Modeling to Study the Effects of Partial Shading on PV Array Characteristics", IEEE Transactions on Energy Conversion, Vol.23, No.1, Mar. 2008.

[3] E.V. Paraskevadaki and A. Papathanassious, "Evaluation of MPP Voltage and Power of mc-Sci PV Modules in Partial Shading Conditions", IEEE Transactions on Energy Conversion, Vol.26, No.3, Sep. 2011.

[4] S. Lyden, M.E. Haque, A. Gargoom, M. Negnevitsky, "Review of Maximum Power Point Tracking Approaches Suitable of PV Systems Under Partial Shading Conditions", Australasian Universities Power Engineering Conference, AUPEC 2013, Hobart, TAS, Australia, Sep.Oct. 2013.

[5] H.A. Sher, A.F. Murtaza, A. Norman, K.E. Addoweesh, K. Al-Haddad, M. Chiaberge, "A New Sensorless Hybrid MPPT Algorithm Based on Fractional Short-Circuit Measurement and P\&O MPPT", IEEE Transactions on Sustainable Energy, IEEE 1949-3029, 2015.
[6] K. Chinranjeevi and Y.S. Rao, "Wind Energy Conversion Systems for Hybrid Algorithm of MPPT", Anveshana's International Journal of Research in Engineering and Applied Sciences, Vol.1, Iss.2, Feb. 2016.

[7] V. Salas, E. Olas, A. Barrado, and A. Lzaro, "Review of the maximum power point tracking algorithms for stand-alone photovoltaic systems," Solar Energy Mater. Solar Cells, vol. 90, no. 11, pp. 1555-1578, 2006. 